\title{
Education, Training and Library Occupations
}

National Cancer Institute

\section{Source}

National Cancer Institute. Education, Training and Library Occupations. NCI Thesaurus.

Code C97631.

A class of professional or vocational positions of employment that involve education, training or libraries. 